\title{
Lytic Bone Lesion: An Unusual Presentation of Hairy Cell Leukemia
}

\author{
Sydney M. Fasulo ${ }^{1}$, Spandana Narvaneni ${ }^{2}$, Vinod Kumar ${ }^{1}$, Anusha Manje Gowda ${ }^{1}$, Yasmeen Sultana ${ }^{1}$ \\ 1. Hematology/Oncology, St. Joseph's University Medical Center, Paterson, USA 2. Internal Medicine, St. Joseph's \\ University Medical Center, Paterson, USA
}

Corresponding author: Sydney M. Fasulo, sydneyfasulo@gmail.com

\begin{abstract}
Hairy cell leukemia (HCL) is a seldom encountered malignancy of lymphocytes with a low incidence in the United States. HCL generally follows an indolent course and not all patients require treatment. Most patients are asymptomatic at the time of diagnosis. Treatment is reserved for those with anemia, thrombocytopenia, neutropenia, recurrent infections, symptomatic splenomegaly, or lymphadenopathy impairing vital organ function. Purine analogs are the mainstay of treatment with a durable response. We report a case of a 49year old Ukrainian male who presented with bone pain secondary to a lytic bone lesion who was diagnosed with HCL.
\end{abstract}

Categories: Radiology, Oncology, Hematology

Keywords: massive splenomegaly, hairy cell leukemia, cladribine, moxetumomab pasudotox, rituximab

\section{Introduction}

Hairy cell leukemia (HCL ) is a rare malignant lymphoproliferative disorder that is often asymptomatic but may present with cytopenia and splenomegaly due to circulating abnormal B-cells. It was first identified by Bouroncle et al. [1] in 1958 and has an estimated incidence, in the United States, of three cases per million persons per year, which equates to approximately 1000 new cases each year [2]. The abnormal B-cells are characterized by a central nucleus, thin cytoplasmic projections resembling hair, and the expression of CD11c, CD25, CD103, CD123, CD20, CD22, CD52, and mild expression of cyclin D [3].

\section{Case Presentation}

We report a case of a 49-year old Ukrainian male with a past medical history of HCL treated seven years prior in Ukraine with cladribine chemotherapy. The patient presented to the emergency department with chest, left shoulder, and right hip pain for three weeks duration. He reported two to three months of increasing fatigue leading to progressive weakness over the week prior. He is a life-long non-smoker and consumes minimal alcohol. Denies fever, chills, night sweats, weight loss, or abdominal pain. He moved from Ukraine to the United States one year prior and denied Chernobyl exposure.

Review began 12/11/2020 Review ended 01/25/2021 Published 01/28/2021

\section{○ Copyright 2021}

Fasulo et al. This is an open access article distributed under the terms of the Creative Commons Attribution License CC-BY 4.0., which permits unrestricted use, distribution, and reproduction in any medium, provided the original author and source are credited.
In the emergency department, the patient was found to have a temperature of 37.2 degrees Celsius, heart rate of 111 beats/min, blood pressure of $148 / 88 \mathrm{mmHg}$, respiratory rate of $16 / \mathrm{min}$. His initial complete blood count demonstrated a white blood cell count of 1.2x109/L (normal range: $4.5-11.0 \times 109 / \mathrm{L}$ ), lymphocyte count of $84 \times 103 / \mathrm{mm} 3$ (normal range: $24.0-44.0 \times 103 / \mathrm{mm} 3$ ), hemoglobin $4.2 \mathrm{~g} / \mathrm{dL}$ (normal range: $13.5-17.5$ $\mathrm{g} / \mathrm{dL}$ ), hematocrit 13.9\% (normal range 41.0-53.0\%), platelets 32,000 $\mu \mathrm{L}$ (normal range: 140,000-440,000 $\mu \mathrm{L}$ ), absolute neutrophil count $190 \mathrm{~mm} 3$ (normal range: 1300-7800 mm3). The Hepatitis panel and COVID-19 tests were all negative. Physical examination showed conjunctival pallor and splenomegaly extending to the umbilicus.

Further laboratory investigation revealed a soluble IL-2 receptor level $>20,000 \mathrm{pg} / \mathrm{mL}$ (normal range 622 $1619 \mathrm{pg} / \mathrm{mL}$ ). Multiple myeloma workup was negative. Imaging revealed splenomegaly measuring $25 \mathrm{~cm}$ on ultrasonography. Computer tomography angiography (CTA) of the chest showed mediastinal adenopathy (Figure 1), and computer tomography of the pelvis showed an increased signal in the right sacrum (Figure 2). 


\section{Cureus}
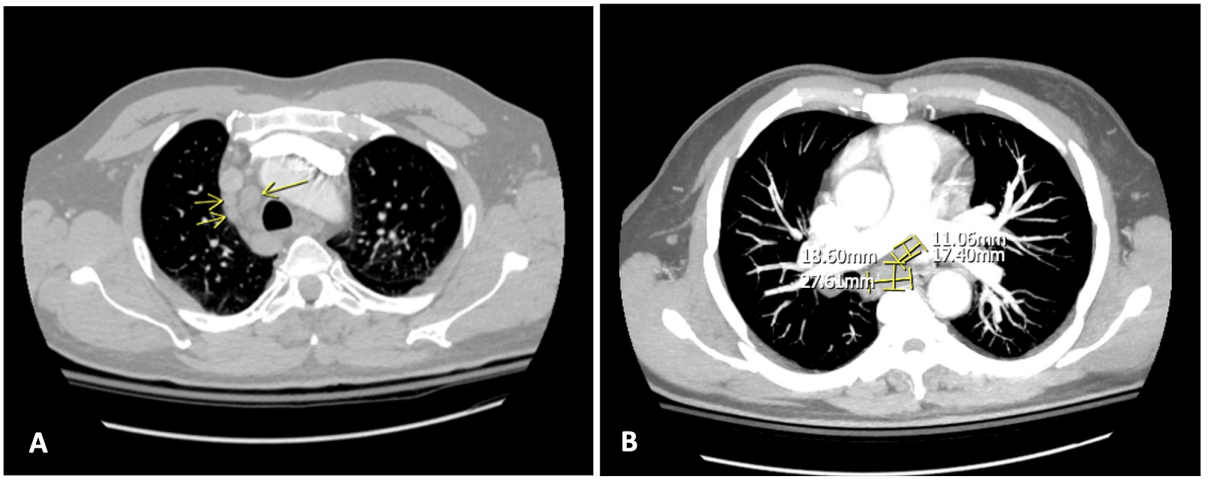

FIGURE 1: Axial images ( $A$ and $B$ ) from a CTA demonstrate mediastinal adenopathy with the largest node measuring $27.6 \times 18.6 \mathrm{~mm}$ in the subcarinal region.

CTA: computer tomography angiography
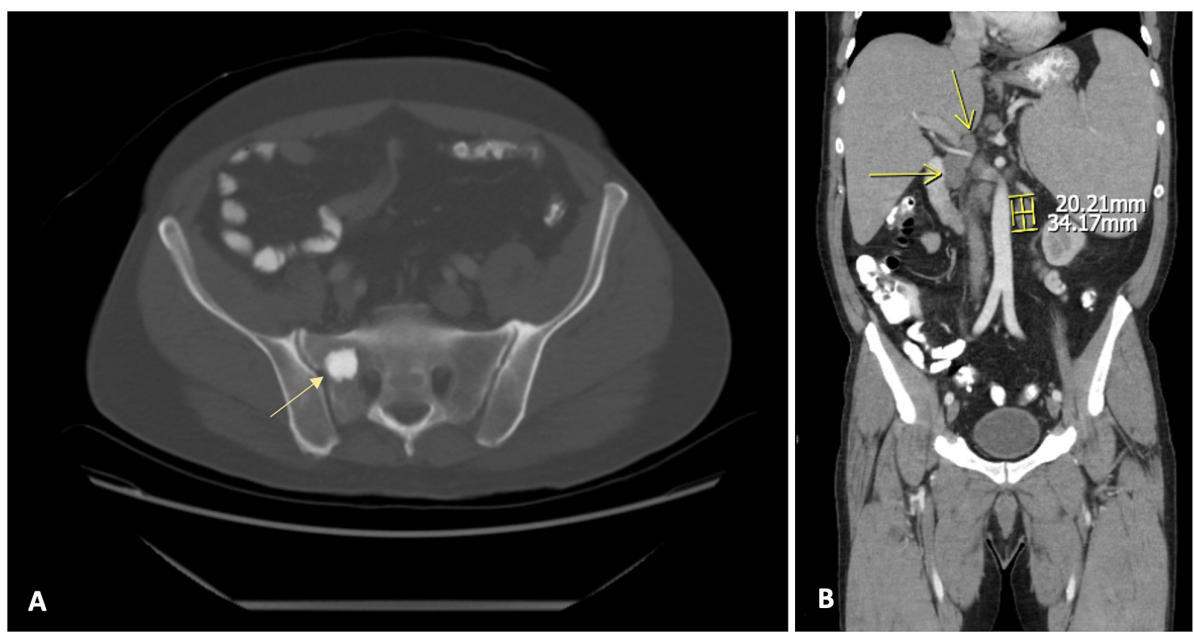

FIGURE 2: An axial image (A) from a CT pelvis shows an increased signal in the right sacrum and the coronal image (B) showing periportal and retroperitoneal lymph nodes suspicious for lymphoid neoplasm.

There was no evidence of metastasis on the bone scan. A bone marrow biopsy was performed initially revealing a dry tap. Aspiration was subsequently achieved and analysis showed complete maturation of myeloid and erythroid populations, no megakaryocytes, and no increase in blasts or plasma cells. The marrow was found to be hypercellular with extensive involvement by atypical lymphocytes which had replaced $>90 \%$ of marrow cellularity. The B-cell population was $18 \%$. Flow cytometry showed a monoclonal population of kappa, CD103, CD25, CD11c, and CD10 positive B-cells. Subsequent testing was positive for BRAF mutation. The bone marrow smear showed small to mildly enlarged atypical lymphoid cells with round to slightly irregular nuclear contour with considerable cytoplasm (Figure 3).
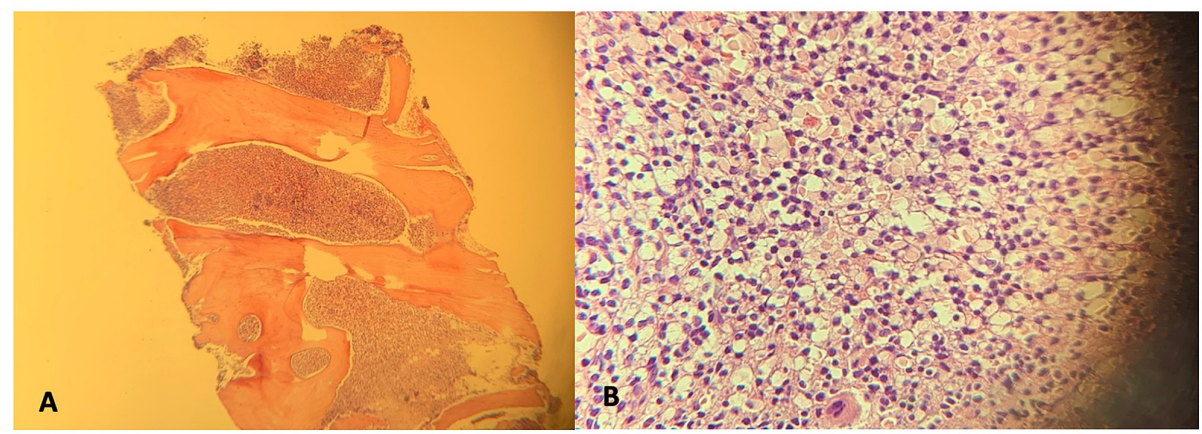


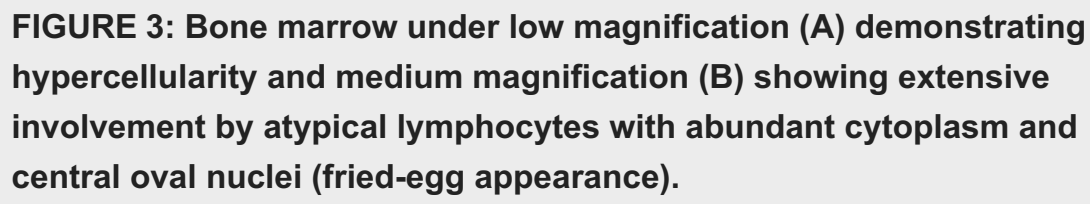

The patient was previously treated with five days of cladribine in Ukraine. As the patient had relapsed seven years after initial treatment, National Comprehensive Cancer Network (NCCN) guidelines dictated that he could be treated again with the same purine analog. We treated the patient with cladribine and concurrent rituximab followed by weekly maintenance rituximab. At the time of this case report submission, the response to treatment has been favorable with near resolution of splenomegaly, improvement in fatigue, and resolution of bone pain.

\section{Discussion}

HCL is a lymphoid malignancy characterized by the increased neoplastic proliferation of small mature B cells with abundant cytoplasm. The cytoplasm has characteristic circumferential, thin, cytoplasmic projections which can be seen in both the bone marrow, peripheral blood, and splenic red pulp. These abnormal hairy cells can become sequestered in the spleen which results in splenomegaly and varying degrees of cytopenia. The cytopenia in HCL can vary from mild to severe and may affect the production of red blood cells, platelets, mature granulocytes, and monocytes. Routine blood work showing anemia, thrombocytopenia, and pancytopenia may initially raise suspicion for HCL in asymptomatic patients while more overt findings such as bleeding or infection may be the presenting signs in symptomatic patients [4]. An article in Blood Research from 2020 compiled atypical presentations of HCL citing case reports of absent splenomegaly, arthralgia, skull lesions, epidural mass with radiculopathy, and a femur lesion [5].

Two types of HCL have been described, the first being classical hairy cell leukemia (HCLc) and the second, variant hairy cell leukemia (HCLv) [6]. HCLv is a chronic B cell lymphoid neoplasm previously thought to be a subtype of HCL but now considered an entity that is distinct from HCLc. HCLv is more aggressive and has a poor response to standard HCL treatment [7].

The initial laboratory evaluation of HCL includes a complete blood count with differential (CBC w/dif), a peripheral blood smear, comprehensive metabolic panel (CMP), electrolytes, uric acid, lactate dehydrogenase (LDH), CD4 count, HIV and hepatitis panel. If HCL is suspected, along with routine labs, a peripheral blood flow should be ordered to confirm the immunophenotyping of circulating mononuclear nuclear cells [6]. A bone marrow aspiration and biopsy should be performed as soon as possible for a definitive diagnosis. The bone marrow aspiration may be a dry tap because of the development of fibrosis. Imaging is not part of the diagnostic criteria for HCL but can be helpful to document splenomegaly and lymphadenopathy.

Most patients with HCL achieve durable remission with purine analog treatment with pentostatin or cladribine and rituximab $[8,9]$. The use of interferon therapy and splenectomy are no longer recommended treatments [10]. The response to treatment can be monitored with a physical examination of spleen size and a CBC w/dif. A bone marrow biopsy has prognostic value and, although not required, should be done four to six months after cladribine treatment, or after normalization of cell counts on CBC w/dif if treatment is with pentostatin.

The goal of treatment is complete remission (CR). CR is defined as normalization of peripheral blood counts (hemoglobin $>11 \mathrm{~g} / \mathrm{dL}$ ), platelets $(>100,000 / \mu \mathrm{L})$ ), absolute neutrophil count $(>1500 / \mu \mathrm{L})$, absence of morphologic evidence of HCL on both peripheral blood smear and bone marrow smear, and regression of splenomegaly - although radiographic documentation of spleen size is not necessary [6]. Minimal residual disease (MRD) is defined as HCL infiltrates recognizable only using polymerase chain reaction testing but not identifiable with traditional immunohistochemical (IHC) evaluation or with flow cytometry. Depending on the criteria used, 13 to 53 percent of patients in apparent CR have evidence of MRD, which may or may not predict future relapse [11-14].

The majority of patients treated with purine analogs will respond; up to $20 \%$ will achieve partial remission and up to $4 \%$ will be classified as a resistant disease and have stable or progressive disease [15]. Patients with a relapse of HCL should be treated based on the timing of the relapse. Relapse within two years of initial treatment can be treated with an alternative purine analog, while patients who relapse after two years can be treated with the same purine analog used previously and the addition of rituximab [6]. Patients with HCL resistance to two or more therapies are candidates for either moxetumomab pasudotox, vemurafenib with rituximab, or bendamustine with rituximab [16].

Moxetumomab pasudotox is an anti-CD22 antibody that has shown good response rates in patients with previously treated HCL but has not been effective in HCLv. A multicenter study evaluated moxetumomab 
pasudotox in patients with either relapsed or refractory HCL who had failed treatment with at least two prior therapies, including at least one purine nucleoside analog [17-19]. Administration of this therapy is complicated and requires hydration, premedication, and monitoring for renal toxicity, electrolyte abnormalities, capillary leak syndrome, and hemolytic uremic syndrome. Long-term follow up is still needed to further evaluate the durability of remission and long-term side effects. Moxetumomab pasudotox is currently approved by the US Food and Drug Administration for adults with relapsed or refractory HCL who received at least two prior therapies, including treatment with a purine nucleoside analog [19].

\section{Conclusions}

HCL can present with a variety of symptoms ranging from asymptomatic to pancytopenia and massive splenomegaly. A routine CBC is valuable in the detection of HCL, as it is most often asymptomatic at diagnosis. Patients presenting with pancytopenia or cytopenia with splenomegaly should be worked up for HCL. Not all patients with HCL require treatment. Treatment indications include anemia (hemoglobin $<10$ $\mathrm{g} / \mathrm{dL}$ ), thrombocytopenia $(<100,000 \mu \mathrm{L})$, neutropenia (WBC $>1500 / \mu \mathrm{L})$, recurrent infections, symptomatic splenomegaly or symptomatic lymphadenopathy. The first-line treatment for HCL includes purine analogs and rituximab with a durable response. Hairy cell Leukemia can have bone lesions and may present with bone pain as we have seen in this case study. A peripheral smear is helpful in the diagnosis of HCL although, as demonstrated here, not all patients will have characteristic hairy cells on peripheral smear. Therefore, IHC is crucial to the diagnosis of HCL.

\section{Additional Information \\ Disclosures}

Human subjects: Consent was obtained or waived by all participants in this study. Conflicts of interest: In compliance with the ICMJE uniform disclosure form, all authors declare the following: Payment/services info: All authors have declared that no financial support was received from any organization for the submitted work. Financial relationships: All authors have declared that they have no financial relationships at present or within the previous three years with any organizations that might have an interest in the submitted work. Other relationships: All authors have declared that there are no other relationships or activities that could appear to have influenced the submitted work.

\section{Acknowledgements}

We would like to express our appreciation to Mehandar Kumar, MD, for his treatment of the patient mentioned in this study and Lan Wang, MD, for her pathologic evaluation and photographic contributions.

\section{References}

1. Bouroncle BA, Wiseman BK, Doan CA: Leukemic reticuloendotheliosis. Blood. 1958, 13:609-630. 10.1182/blood-2016-01-696179

2. Morton LM, Wang SS, Devesa SS, Hartge P, Weisenburger DD, Linet MS: Lymphoma incidence patterns by WHO subtype in the United States, 1992-2001. Blood. 2006, 107:265-276. 10.1182/blood-2005-06-2508

3. Matutes E: Immunophenotyping and differential diagnosis of hairy cell leukemia . Hematol Oncol Clin North Am. 2006, 20:1051-1063. 10.1016/i.hoc.2006.06.012

4. Grever MR: How I treat hairy cell leukemia. Blood. 2010, 115:21-28. 10.1182/blood-2009-06-195370

5. Pattnaik SA, Padhi S, Chhabra G, Panigrahi MK, Das PK, Bhola RK, Mishra S: Atypical presentation of hairy cell leukemia: a report and comprehensive review. Blood Res. 2020, 55:123-127. 10.5045/br.2020.2020069

6. Grever MR, Abdel-Wahab O, Andritsos LA, et al.: Consensus guidelines for the diagnosis and management of patients with classic hairy cell leukemia. Blood. 2017, 129:553-560. 10.1182/blood-2016-01-689422

7. Robak T: Hairy-cell leukemia variant: recent view on diagnosis, biology and treatment. Cancer Treat Rev. 2011, 37:3-10. 10.1016/j.ctrv.2010.05.003

8. Dearden CE, Else M, Catovsky D: Long-term results for pentostatin and cladribine treatment of hairy cell leukemia. Leuk Lymphoma. 2011, 52:21-24. 10.3109/10428194.2011.565093

9. Ravandi F, O’Brien S, Jorgensen J, et al.: Phase 2 study of cladribine followed by rituximab in patients with hairy cell leukemia. Blood. 2011, 118:3818-3823. 10.1182/blood-2011-04-351502

10. Federico M, Frassoldati A, Lamparelli T, et al.: Long-term results of alpha interferon as initial therapy and splenectomy as consolidation therapy in patients with hairy cell leukemia. Final report from the Italian Cooperative Group for HCL. Ann Oncol. 1994, 5:725-731. 10.1093/oxfordjournals.annonc.a058977

11. Ellison DJ, Sharpe RW, Robbins BA, Spinosa JC, Leopard JD, Saven A, Piro LD: Immunomorphologic analysis of bone marrow biopsies after treatment with 2-chlorodeoxyadenosine for hairy cell leukemia. Blood. 1994, 84:4310-4315.

12. Hakimian D, Tallman MS, Kiley C, Peterson L: Detection of minimal residual disease by immunostaining of bone marrow biopsies after 2-chlorodeoxyadenosine for hairy cell leukemia. Blood. 1993, 82:1798-1802.

13. Wheaton S, Tallman MS, Hakimian D, Peterson L: Minimal residual disease may predict bone marrow relapse in patients with hairy cell leukemia treated with 2-chlorodeoxyadenosine. Blood. 1996, 87:15561560 .

14. Sigal DS, Sharpe R, Burian C, Saven A: Very long-term eradication of minimal residual disease in patients with hairy cell leukemia after a single course of cladribine. Blood. 2010, 115:1893-1896. 10.1182/blood2009-10-251645

15. Else M, Dearden CE, Matutes E, et al.: Long-term follow-up of 233 patients with hairy cell leukaemia, 


\section{Cureus}

treated initially with pentostatin or cladribine, at a median of 16 years from diagnosis. Br J Haematol. 2009, 145:733-740. 10.1111/j.1365-2141.2009.07668.x

16. Tiacci E, Park JH, De Carolis L, et al.: Targeting mutant BRAF in relapsed or refractory hairy-cell leukemia . N Engl J Med. 2015, 373:1733-1747. 10.1056/NEJMoa1506583

17. Kreitman RJ, Tallman MS, Robak T, et al.: Phase I trial of anti-CD22 recombinant immunotoxin moxetumomab pasudotox (CAT-8015 or HA22) in patients with hairy cell leukemia. J Clin Oncol. 2012, 30:1822-1828. 10.1200/JCO.2011.38.1756

18. Kreitman RJ, Tallman MS, Robak T, et al.: Minimal residual hairy cell leukemia eradication with moxetumomab pasudotox: phase 1 results and long-term follow-up. Blood. 2018, 131:2331-2334. 10.1182/blood-2017-09-803072

19. Kreitman RJ, Dearden C, Zinzani PL, et al.: Moxetumomab pasudotox in relapsed/refractory hairy cell leukemia. Leukemia. 2018, 32:1768-1777. 10.1038/s41375-018-0210-1 